Homology, Homotopy and Applications, vol. 19(1), 2017, pp.23-35

\title{
BIKEI HOMOLOGY
}

\author{
SAM NELSON AND JAKE ROSENFIELD
}

(communicated by John R. Klein)

\begin{abstract}
We introduce a modified homology and cohomology theory for involutory biquandles (also known as bikei). We use bikei 2cocycles to enhance the bikei counting invariant for unoriented knots and links as well as unoriented and non-orientable knotted surfaces in $\mathbb{R}^{4}$.
\end{abstract}

\section{Introduction}

In [11], Joyce introduced an algebraic structure known as quandles which can be used to define computable invariants of oriented knots and links (see also [18]). For unoriented knots and links, a special case known as involutory quandles or kei has been studied going back to Takasaki [22]. In [9] quandles were generalized to racks and in $[\mathbf{1 0}]$ racks were generalized to biracks. In $[\mathbf{1}]$, the involutory case of biquandles was considered, now known as bikei.

In [10] a homology theory for racks and biracks was introduced in which the 2-cocycle condition corresponds to the Reidemeister III move for a certain way of associating 2-chains to crossings in an oriented rack-colored knot or link diagram. In [3] a subcomplex was defined corresponding to Reidemeister I moves in the quandle case, leading to the theory of quandle 2-cocycle invariants of knots and links. In [2] this construction was generalized to the biquandle case. In [7] the degenerate subcomplex was generalized for the case of non-quandle racks, in each case defining a new family of cocycle enhancements of counting invariants.

In this paper we introduce a generalization of biquandle homology to the case of bikei which we call bikei homology. The paper is organized as follows. In Section 2 we review the basics of bikei and the bikei counting invariant. In Section 3 we introduce bikei homology and cohomology. In Section 4 we define the bikei cocycle enhancements of the bikei counting invariant for unoriented knots and provide some examples. In Section 5 we extend the bikei cocycle invariant to unoriented (including non-orientable) knotted surfaces in $\mathbb{R}^{4}$. In Section 6 we finish with some questions for future work.

Partially supported by Simons Foundation collaboration grant 316709 .

Received March 10, 2016, revised May 13, 2016; published on March 8, 2017.

2010 Mathematics Subject Classification: 57M27, 57M25.

Key words and phrases: bikei, involutory biquandle, cocycle invariant, bikei homology.

Article available at http://dx.doi.org/10.4310/HHA.2017.v19.n1.a2

Copyright (C) 2017, Sam Nelson and Jake Rosenfield. Permission to copy for private use granted. 


\section{Bikei}

We begin with a review of bikei (see $[\mathbf{1}, \mathbf{8}]$ for more).

Definition 2.1. A bikei is a set $X$ with two binary operations $\underline{*}, \bar{*}: X \times X \rightarrow X$ satisfying for all $x, y, z \in X$

(i) $x \underline{*} x=x \bar{*} x$,

(ii)

$$
\begin{aligned}
& (x \bar{*} y) \bar{*} y=x \\
& (x \underline{*} y) \underline{*} y=x \quad \text { (ii.ii), } \\
& x \underline{*}(y \bar{*} x)=x \text { 米 } y \quad \text { (ii.iii), } \\
& x \bar{*}(y \underline{*} x)=x \bar{*} y \quad \text { (ii.iv), }
\end{aligned}
$$

and

(iii) (Exchange Laws)

$$
\begin{aligned}
& (x \bar{*} y) \bar{*}(x \bar{*} y)=(x \bar{*} z) \bar{*}(y \underline{*} z) \quad \text { (iii.i), } \\
& (x \underline{*} y) \bar{*}(x \underline{*} y)=(x \bar{*} z) \underline{*}(y \bar{*} z) \quad \text { (iii.ii), } \\
& (x \underline{*} y) \underline{*}(z \underline{*} y)=(x \underline{*} z) \underline{*}(y \bar{*} z) \quad \text { (iii.iii). }
\end{aligned}
$$

Note that $x \underline{*} y$ and $x \bar{*} y$ are also denoted in the literature by $x^{y}=B_{2}(x, y)$ and $x_{y}=B_{1}(x, y)$ respectively; we are following the notation used in $[\mathbf{8}]$.

Some standard examples (see $[\mathbf{1}, \mathbf{8}]$ ) of bikei structures include:

Example 2.2. Let $X$ be a set and $\sigma: X \rightarrow X$ any involution, i.e., any map such that $\sigma^{2}=\operatorname{Id}_{X}$. Then $X$ is a bikei with operations

$$
x \underline{*} y=x \bar{*} y=\sigma(x)
$$

known as a constant action bikei.

Example 2.3. Let $\Lambda=\mathbb{Z}[t, s] /\left(t^{2}-1, s^{2}-1,(t-1)(s-1)\right)$ be the quotient of the ring of two-variable polynomials with integer coefficients such that $s^{2}=t^{2}=1$ by the ideal generated by $(1-t)(1-s)$. Then any $\Lambda$-module $X$ is a bikei with operations

$$
x \underline{*} y=t x+(s-t) y, \quad x \bar{*} y=s x
$$

known as an Alexander bikei. To see this, we can verify the axioms; we will show (iii) and leave the verification of (i) and (ii) to the reader.

$$
\begin{aligned}
(x \bar{*} y) \bar{*}(x \bar{*} y) & =s(s x) \\
& =(x \bar{*} z) \bar{*}(y \text { * } z), \\
(x \underline{*} y) \bar{*}(x \underline{*} y) & =s(t x+(s-t) y) \\
& =t(s x)+(s-t)(s y) \\
& =(x \bar{*} z) \text { * }(y \bar{*} z), \\
(x \underline{*} y) \underline{*}(z \underline{*} y) & =t(t x+(s-t) y)+(s-t)(t z+(s-t) y) \\
& =t^{2} x+t(s-t) y+t(s-t) z+(s-t)^{2} y \\
& =t^{2} x+t(s-t) z+(s-t)(t+s-t) y \\
& =t(t x+(s-t) z)+(s-t)(s y) \\
& =(x \underline{*} z) \underline{*}(y \bar{*} z) .
\end{aligned}
$$


Definition 2.4. A map $f: X \rightarrow Y$ between bikei is a bikei homomorphism if we have

$$
f(x \underline{*} y)=f(x) \underline{*} f(y) \quad \text { and } \quad f(x \bar{*} y)=f(x) \bar{*} f(y)
$$

for all $x, y \in X$. A bijective bikei homomorphism is a bikei isomorphism.

Example 2.5. Let $X=\left\{x_{1}, \ldots, x_{n}\right\}$ be a finite set. We can represent any bikei structure on $X$ with an $n \times 2 n$ block matrix Mencoding the operation tables of $\underline{*}$ and $*$ by setting $M_{j, k}=l$ and $M_{j, k+n}=m$ where $x_{j} x_{x_{k}}=x_{l}$ and $x_{j} \bar{*} x_{k}=x_{m}$ for $j, k \in$ $\{1, \ldots, n\}$. For example, there are two nonisomorphic bikei on the set $X=\left\{x_{1}, x_{2}\right\}$, given by the matrices

$$
\left[\begin{array}{ll|ll}
1 & 1 & 1 & 1 \\
2 & 2 & 2 & 2
\end{array}\right] \quad \text { and } \quad\left[\begin{array}{ll|ll}
2 & 2 & 2 & 2 \\
1 & 1 & 1 & 1
\end{array}\right]
$$

See $[\mathbf{1}, \mathbf{8}, \mathbf{2 1}]$ for more.

Example 2.6. (See $[\mathbf{1}]$ for more.) Let $D$ be an unoriented knot or link diagram representing an unoriented knot or link $K$ and let $G$ be a set of generators corresponding to semiarcs in $D$. The set $W$ of bikei words in $G$ is defined recursively by the rules

(i) $x \in G \Rightarrow x \in W$ and

(ii) $x, y \in W \Rightarrow x \underline{*} y, x \bar{*} y \in W$.

Then the fundamental bikei of $D$, denoted $\mathcal{B K}(D)$, is the set of equivalence classes of $W$ under the equivalence relation generated by the bikei axioms and the crossing relations in $D$, i.e.,

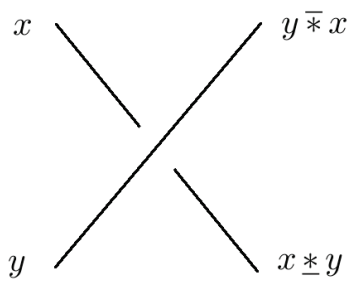

We express such a bikei with a bikei presentation, i.e., an expression of the form

$$
\mathcal{B K}(D)=\left\langle g_{1}, \ldots, g_{n} \mid r_{1}, \ldots, r_{n}\right\rangle,
$$

where $\left\{g_{1}, \ldots, g_{n}\right\}$ are generators and $\left\{r_{1}, \ldots, r_{n}\right\}$ are crossing relations, with the bikei axiom relations understood. It is easy to check that Reidemeister moves on $D$ induce Tietze moves on presentations, and hence the isomorphism type of the fundamental bikei is an invariant of unoriented knots and links; hence, we will generally write $\mathcal{B K}(K)$ instead of $\mathcal{B K}(D)$.

Given an unoriented knot or link $K$ represented by a diagram $D$ and a finite bikei $X$, the bikei counting invariant $\Phi_{X}^{\mathbb{Z}}(K)$ is the cardinality of the set of bikei homomorphisms $f: \mathcal{B K}(K) \rightarrow X$, i.e.,

$$
\Phi_{X}^{\mathbb{Z}}(K)=|\operatorname{Hom}(\mathcal{B K}(K), X)| .
$$

The superscript $\mathbb{Z}$ indicates this is the integer-valued unenhanced version of the invariant; we will soon enhance this invariant with bikei cocycles. 
Every such homomorphism assigns an element of $X$ to each generator of $\mathcal{B K}(K)$, which we can think of as coloring the corresponding semiarc in $D$. Conversely, an assignment of elements of $X$ to the semiarcs in $D$ determines a bikei homomorphism $f: \mathcal{B K}(K) \rightarrow X$ only if it satisfies the crossing relations at every crossing. Hence, we can compute the bikei counting invariant of an unoriented knot or link by counting bikei colorings of any diagram of $D$ which satisfy the crossing relations.

Example 2.7. Consider the bikei $X=\mathbb{Z}_{2}=\{0,1\}$ with $x$ ㄴy $y=x \bar{*} y=x+1$. As a coloring rule, this says that each time we go through a crossing either over or under, we switch from 0 to 1 or 1 to 0 . Then for any classical knot, there are exactly two $X$-colorings, determined by our choice of starting color on a choice of semiarc.
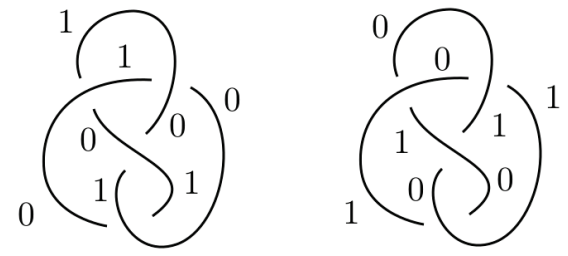

In the next section, we will enhance the bikei counting invariant with cocycles in a bikei homology theory to get a stronger invariant following $[\mathbf{3}, \mathbf{2}]$, etc., but using bikei and unoriented diagrams.

\section{Bikei homology}

Definition 3.1. Let $X$ be a bikei and $A$ and abelian group. Set $C_{n}(X ; A)=A\left[X^{n}\right]$ for $n \geqslant 1$ and $\{0\}$ otherwise. The birack boundary map $\partial_{n}: C_{n}(X ; A) \rightarrow C_{n-1}(X ; A)$ is defined on generators $\vec{x}=\left(x_{1}, \ldots, x_{n}\right)$ by

$$
\partial(\vec{x})=\sum_{k=1}^{n}(-1)^{k-1}\left(\partial_{k}^{1}(\vec{x})-\partial_{k}^{2}(\vec{x})\right),
$$

where

$$
\begin{aligned}
\partial_{k}^{1}\left(x_{1}, \ldots, x_{n}\right) & =\left(x_{1}, \ldots, x_{k-1}, x_{k+1}, \ldots, x_{n}\right) \quad \text { and } \\
\partial_{k}^{2}\left(x_{1}, \ldots, x_{n}\right) & =\left(x_{1} \underline{*} x_{k}, \ldots, x_{k-1} \underline{*} x_{k}, x_{k+1} \bar{*} x_{k}, \ldots, x_{n} \bar{*} x_{k}\right),
\end{aligned}
$$

and extended to $C_{n}(X ; A)$ by linearity. The resulting homology and cohomology groups $H_{n}(X ; A)=\operatorname{Ker} \partial_{n} / \operatorname{Im} \partial_{n-1}$ and $H^{n}=\operatorname{Ker} \delta^{n+1} / \operatorname{Im} \delta^{n}$ where $\delta^{n} \phi=\phi \partial_{n}$ are the birack homology and cohomology groups of $X$ with coefficients in $A$.

In previous work $[\mathbf{3}, \mathbf{2}, \mathbf{6}]$, the subset $C_{n}^{D}(X ; A)$ of $C_{n}(X ; A)$ generated by elements $\left(x_{1}, \ldots, x_{n}\right)$ with $x_{j}=x_{j+1}$ for some $j=1, \ldots, n-1$ was identified as the degenerate subcomplex. Then the biquandle homology and cohomology groups, also known as the Yang-Baxter homology and cohomology groups, are the homology and cohomology groups of the quotient complex $C_{n}^{B}(X ; A)=C_{n}(X ; A) / C_{n}^{D}(X ; A)$.

We now introduce a slight generalization for bikei.

Definition 3.2. Let $X$ be a bikei. The bikei degenerate subgroups of $C_{n}(X ; A)$, denoted $C_{n}^{B D}(X ; A)$, are generated by chains of the form $(x)-(x$ ․y) and 
$(x)-(x \bar{*} y)$ when $n=1$, chains of the forms

$$
(x, x),(x, y)-(x \underline{*} y, y \bar{*} x),(x, y)+(x, y \bar{*} x) \text { and }(x, y)+(x \underline{x} y, y)
$$

for $n=2$ and by chains of the form $(\ldots, x, x, \ldots)$ for $n \geqslant 2$.

Proposition 3.3. For a bikei $X,\left(C_{n}^{B D}, \partial_{n}\right)$ forms a subcomplex.

Proof. When $n=2$ the degenerate chains $(x, y)+(x \underline{*} y, y)$ and $(x, y)+(x, y \bar{*} x)$ have boundary

$$
\begin{aligned}
\partial((x, y)+(x \underline{*} y, y))= & {[(y)-(y \bar{*} x)-(x)+(x \underline{*} y)] } \\
& \quad+[(y)-(y \bar{*}(x \underline{*} y))-(x \underline{*} y)+((x \underline{*} y) \underline{*} y)] \\
= & {[(y)-(y \bar{*} x)-(x)+(x \underline{x} y)]+[(y)-(y \bar{*} x)-(x \underline{*} y)+(x)] } \\
= & 2[(y)-(y \bar{*} x)]
\end{aligned}
$$

and

$$
\begin{aligned}
\partial((x, y)+(x, y \bar{*} x))= & {[(y)-(y \bar{*} x)-(x)+(x \underline{*} y)] } \\
& +[(y \bar{*} x)-((y \bar{*} x) \bar{*} x)-(x)+(x \underline{*}(y \bar{*} x))] \\
= & {[(y)-(y \bar{*} x)-(x)+(x \underline{w})]+[(y \bar{*} x)-(y)-(x)+(x \underline{x})] } \\
= & 2[(x \underline{*} y)-(x)],
\end{aligned}
$$

while the chains $(x, y)-(x * y, y \bar{*} x)$ have boundary

$$
\begin{aligned}
\partial((x, y)-(x \underline{*} y, y \bar{*} x))= & {[(y)-(y \bar{*} x)-(x)+(x \underline{*} y)] } \\
& -[(y \bar{*} x)-((y \bar{*} x) \bar{*}(x \underline{*} y))-(x \underline{*} y)+((x \underline{*} y) \underline{*}(y \bar{*} x))] \\
= & {[(y)-(y \bar{*} x)-(x)+(x \underline{*} y)]-[(y \bar{*} x)-(y)-(x \underline{x})+(x)] } \\
= & 2[(y)-(y \bar{*} x)]-2[(x)-(x \underline{*} y)] \in C_{1}^{B D}(X ; A) .
\end{aligned}
$$

Hence, $\partial_{n}\left(C_{n}^{B D}(X ; A)\right) \subset C_{n-1}^{B D}(X ; A)$ and $\left(\partial, C_{n}^{B D}(X ; A)\right)$ is a subcomplex.

Definition 3.4. Let $X$ be a bikei. Then for each $n \geqslant 1$, set

$$
C_{n}^{B K}(X ; A)=C_{n}(X ; A) / C_{n}^{B D}(X ; A) .
$$

The resulting homology and cohomology groups $H_{n}^{B K}(X ; A)$ and $H_{B K}^{n}(X ; A)$ are the bikei homology and cohomology groups of $X$. We will generally denote elements of $C^{n}(X ; A)$ and their equivalence classes in $H_{B K}^{n}(X ; A)$ by $\phi$.

Since $C_{n}^{B D}(X ; A)=C_{n}^{D}(X ; A)$ for $n \geqslant 3$, we have:

Theorem 3.5. For $n>3$, the biquandle and bikei homology and cohomology groups for a bikei $X$ coincide, i.e., $H_{n}^{B}(X ; A)=H_{n}^{B K}(X ; A)$ and $H_{B}^{n}(X ; A)=H_{B K}^{n}(X ; A)$.

Proposition 3.6. If $X$ is a bikei in which $x \bar{*} y=x$ for all $x, y$ (that is, a kei) or a bikei in which $x \underline{*} y=x$ for all $x, y$, then $H_{B K}^{2}(X ; \mathbb{F})=\{0\}$ for any field $\mathbb{F}$ of characteristic $\neq 2$.

Proof. In either of the listed cases, the degenerate group includes all cochains $\phi \in$ $C^{2}(X ; A)$ of the form $\phi(x, y)+\phi(x, y)=2 \phi(x, y)$, so we must have $2 \phi(x, y)=0$. Then if our coefficients belong to a field of characteristic $\neq 2$, we must have $\phi(x, y)=0$ for all $x, y \in X$. 
At first, it may seem like bikei homology is completely trivial, but it turns to have nontrivial torsion part in at least some cases.

Proposition 3.7. Let $A$ be a commutative ring with identity and let $X$ be an Alexander bikei structure on $A$, i.e., a choice of units $t, s \in A^{\times}$such that $(1-s)(1-t)=0$ defining operations

$$
x \underline{*} y=t x+(s-t) y \quad \text { and } \quad x \bar{*} y=s x .
$$

Then for elements $a, b \in A$ satisfying

$$
b=-a \quad \text { and } \quad 2 a=a(1+s)=a(1+t)=a(1-t)=a(s-t-2)=0,
$$

the linear map $\phi(x, y)=a x+$ by defines a bikei cocycle in $H_{B K}^{2}(X ; A)$.

We will call such a cocycle a linear Mochizuki bikei cocycle since it is similar to Mochizuki cocycles for Alexander quandles [19].

Proof. We check the bikei degeneracy and cocycle conditions. First, checking the degeneracy conditions, $b=-a$ implies $\phi(x, x)=a x+b x=(a+b) x=0$. Then setting $\phi(x, y)=a(x-y)$, the other degeneracy conditions are satisfied:

$$
\begin{aligned}
\phi(x, y)-\phi(x \underline{*} y, y \bar{*} x) & =0, \\
a(x-y)-a(t x+(s-t) y-s y) & =0, \\
a(1-t) x-a(-1+s-t-s) y & =0, \\
a(1-t) x+a(1+t) y & =0
\end{aligned}
$$

since $a(1-t)=a(1+t)=0$,

$$
\begin{aligned}
\phi(x, y)+\phi(x \underline{*} y, y) & =0, \\
a(x-y)+a(t x+(s-t) y-y) & =0 \\
a(1+t) x+a(-1+s-t-1) y & =0 \\
a(1+t) x-a(s-t-2) y & =0
\end{aligned}
$$

since $a(s-t-2)=0$, and

$$
\begin{aligned}
\phi(x, y)+\phi(x, y \bar{*} x) & =0, \\
a(x-y)+a(x-s y) & =0 \\
a(2) x+a(-1-s) y & =0 \\
2 a x-a(1+s) y & =0 .
\end{aligned}
$$

Finally, we check the birack cocycle condition:

$$
\begin{aligned}
\phi(x, y)+\phi(y, z)+\phi(x \text { } y, z \bar{*} y)= & \phi(x, z)+\phi(x \text { * } z, y \text { * } z)+\phi(y \bar{*} x, z \bar{*} x), \\
a(x-y)+a(y-z)+a(t x+(s-t) y-s z)= & a(x-z)+a(t x+(s-t) z \\
& -t y-(s-t) z)+a(s y-s z), \\
a(1-t) x+a(s-t) y-a(1+s) z= & a(1+t) x+a(s-t) y-a(1+s) z
\end{aligned}
$$

as required. 
Example 3.8. Let $X=\mathbb{Z}_{8}$ and set $s=3, t=1$ and $a=4$. Then we have $(1-s)(1-$ $t)=0$, so $X$ is a bikei with operations

$$
x \underline{*} y=x+2 y \quad \text { and } \quad x \bar{*} y=3 x .
$$

Then we verify that

$$
2(4)=4(1+3)=4(1+1)=4(1-1)=4(3-1-2)=0
$$

and $\phi(x, y)=4 x-4 y$ is a nonzero bikei cocycle.

\section{Cocycle enhancements}

Our motivation for bikei homology comes from the desire to extend cocycle enhancements of the bikei counting invariant to unoriented knots and links, and, in particular, to non-orientable knotted surfaces in $\mathbb{R}^{4}$.

Let $\phi \in H_{B K}^{2}(X ; A)$ and let $D$ be an unoriented knot or link diagram representing a knot or link $K$. For any bikei homomorphism $f: \mathcal{B K}(K) \rightarrow X$, let $D_{f}$ denoted the $X$-coloring of $D$ determined by $f$. That is, $f$ is a bikei homomorphism assigning elements of $X$ to generators of $\mathcal{B K}(K)$ (which correspond one to one with semiarcs in $D$ ), while $D_{f}$ is the knot diagram $D$ with semiarcs labeled with their images $f\left(x_{j}\right) \in X$. Then at each crossing we assign a Boltzmann weight $\phi(x, y)$ where $x$ and $y$ are the bikei colors on the under and over crossing semiarcs when the crossing is positioned as depicted, with the overstrand going from upper right to lower left.

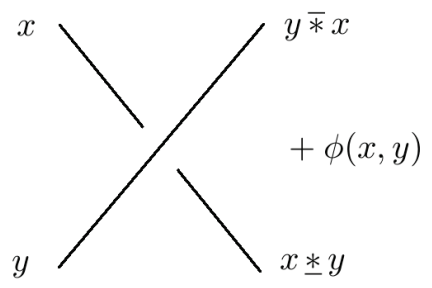

Then the Boltzmann weight for the bikei coloring $f$ is the sum of the Boltzmann weights $\phi(x, y)$ at each crossing $C$ in the set $\mathcal{C}\left(D_{f}\right)$ of crossings in diagram $D_{f}$,

$$
B W(f)=\sum_{C \in \mathcal{C}\left(D_{f}\right)} \phi(x, y) .
$$

The bikei 2-cocycle conditions are precisely the conditions required to ensure that the Boltzmann weight is unchanged by Reidemeister moves. The degeneracy conditions insure that the Boltzmann weight is well-defined and give invariance under Reidemeister I and II moves:
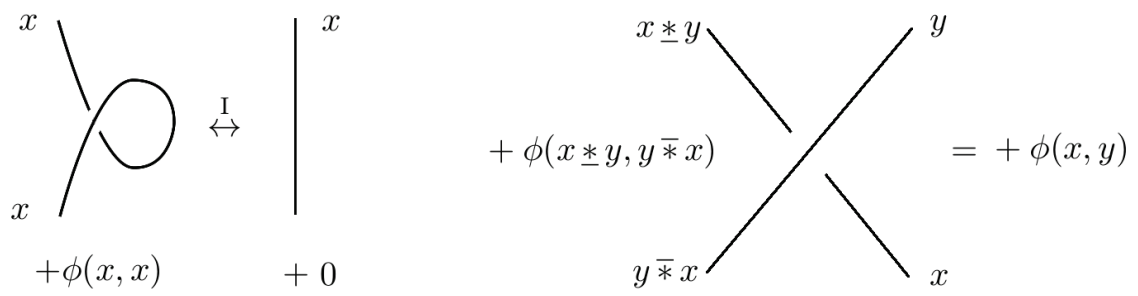


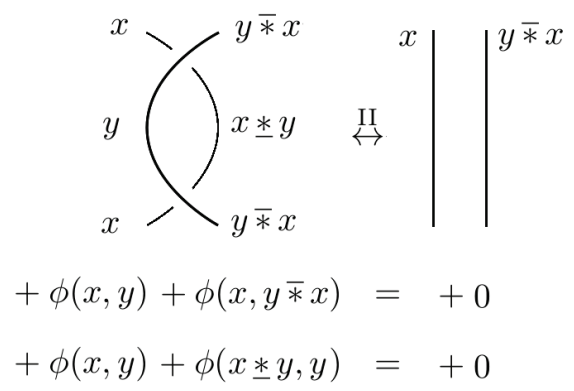

The 2-cocycle condition

$$
\begin{aligned}
\delta^{2}(\phi(x, y, z)) & =\phi\left(\partial_{2}(x, y, z)\right) \\
& =\phi(-(y, z)+(x \text { ㄴ } y, z \bar{*} y)+(x, z)-(y \bar{*} x, z \bar{*} x)-(x, y)+(x \text { ․ } y, y \underline{*} z)) \\
& =0
\end{aligned}
$$

guarantees equivalence under Reidemeister III moves:
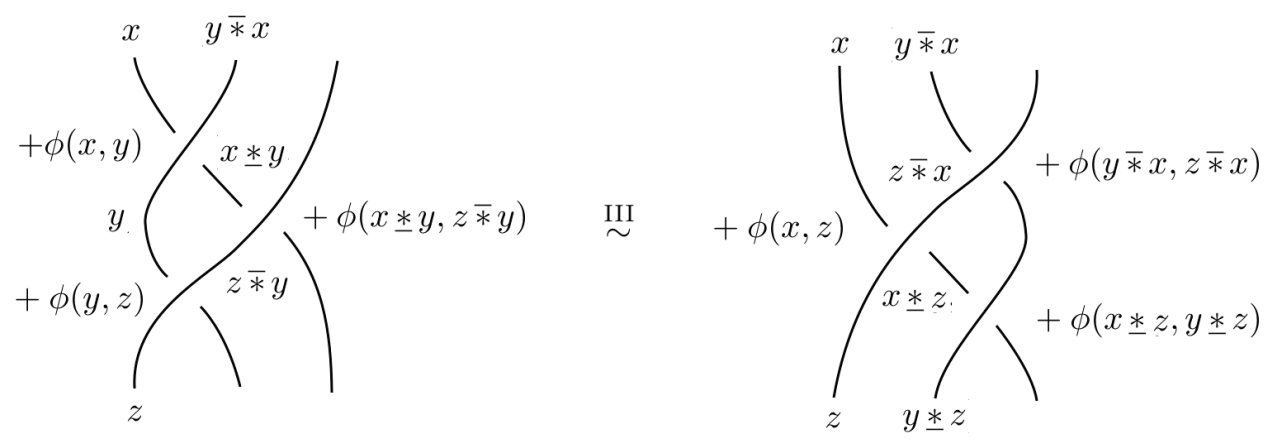

Thus, we have

Definition 4.1. Let $X$ be a finite bikei and $\phi \in H_{B K}^{2}(X ; A)$. Then for any unoriented knot or link $K$ represented by a diagram $D$, the 2-cocycle enhanced bikei counting invariant of $K$ is the multiset of Boltzmann weights

$$
\Phi_{X}^{\phi, M}(D)=\left\{B W\left(D_{f}\right) \in A \mid f \in \operatorname{Hom}(\mathcal{B K}(K), X)\right\} .
$$

We can convert this multiset into a polynomial form for ease of comparison by making each Boltzmann weight a formal exponent of a dummy variable $u$ and converting multiplicities to positive integer coefficients:

$$
\Phi_{X}^{\phi}(D)=\sum_{f \in \operatorname{Hom}(\mathcal{B K}(K), X)} u^{B W\left(D_{f}\right)} .
$$

By construction (and also see $[\mathbf{2}]$ etc.), we have

Theorem 4.2. If $X$ is a finite bikei, $\phi \in H_{B K}^{2}(X)$ and $D$ and $D^{\prime}$ are unoriented knot or link diagrams related by Reidemeister moves, then

$$
\Phi_{X}^{\phi, M}(D)=\Phi_{X}^{\phi, M}\left(D^{\prime}\right) \quad \text { and } \quad \Phi_{X}^{\phi}(D)=\Phi_{X}^{\phi}\left(D^{\prime}\right) .
$$


As with many knot invariants, we can extend $\Phi_{X}^{\phi}$ to the case of virtual knots and links by simply ignoring the virtual crossings, thinking of our virtual knot diagram as drawn on a surface with sufficient genus to avoid virtual crossings; see $[\mathbf{1 2}, \mathbf{4}]$ for more. In particular, semiarcs do not end at virtual crossings.

Example 4.3. Let $X$ be the Alexander bikei from Example 3.8 and consider the virtual Hopf link $V H$ below.

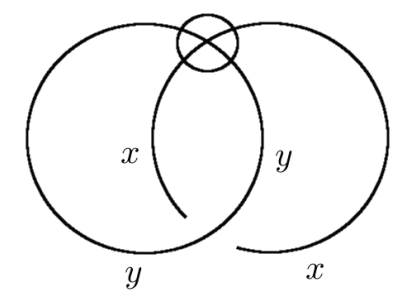

We obtain system of coloring equations

$$
\begin{aligned}
x * * * y & =x \\
y * x & =y
\end{aligned} \Longrightarrow \begin{aligned}
x+2 y & =x \\
3 y & =y
\end{aligned} \quad \Longrightarrow \quad 2 y=0,
$$

so a pair $(x, y)$ yields a valid coloring for $y \in\{0,4\}$ and no further conditions on $x \in \mathbb{Z}_{8}$, so there are $16 X$-colorings. Each coloring has a Boltzmann weight of

$$
\phi(x, y)=4(x-y)= \begin{cases}4 & x \text { odd } \\ 0 & x \text { even }\end{cases}
$$

at the single crossing, so the invariant is $\Phi_{X}^{\phi}(V H)=8+8 u^{4}$.

We can compare this with the case of the usual Hopf link

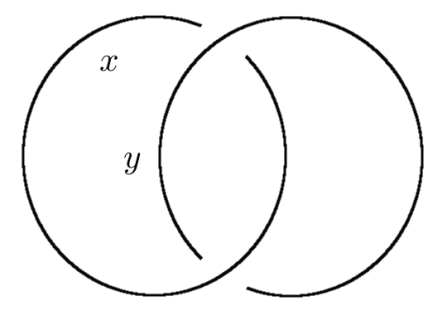

where we obtain coloring equations $3 x=x+2 y$ and $3 y=y+2 x$, both reducing to $2 x=2 y$. Then there are sixteen colorings, and each has Boltzmann weight $\phi(x, y)+$ $\phi(y, x)=4(x-y)+4(y-x)=0$, yielding an invariant value of $\Phi_{X}^{\phi}(H)=16$. Now, the virtual Hopf link can be distinguished from the classical Hopf link in other ways, e.g. the Hopf link has two colorings by the bikei $\mathbb{Z}_{2}$ with $x$ ㅊy $=x \bar{*} y=x+1$ while the virtual Hopf link has none; this example demonstrates that $\Phi_{X}^{\phi}$ is not determined by the counting invariant and hence is a proper enhancement.

\section{Invariants of knotted surfaces}

We can also define bikei cocycle invariants for knotted unoriented (including nonorientable) surfaces in $\mathbb{R}^{4}$ in the same way. Recall that a marked graph diagram, also 
called a marked vertex diagram, is a diagram with ordinary crossings together with saddle crossings
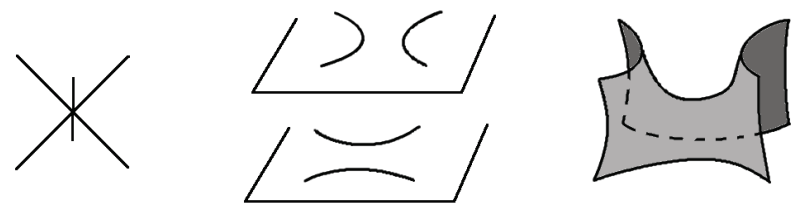

representing saddle point. More precisely, given a knotted surface $\Sigma \subset \mathbb{R}^{4}$, we move the maxima in the $x_{4}$ direction to the hyperplane $x_{4}=1$, the minima to $x_{4}=-1$ and the saddle points to $x_{4}=0$. Then the intersection of $\Sigma$ with $x_{4}=0$ is a link diagram with singularities at the saddle points; we indicate the direction of the saddle with a small bar. Such a diagram represents a knots closed surface if both resolutions of the saddle yield unlinks; otherwise, the diagram represents a cobordism between the links represented by the smoothed diagrams. Two such diagrams represent ambient isotopic knotted surfaces if and only if they are related by a sequence of the Reidemeister moves together with the Yoshikawa moves
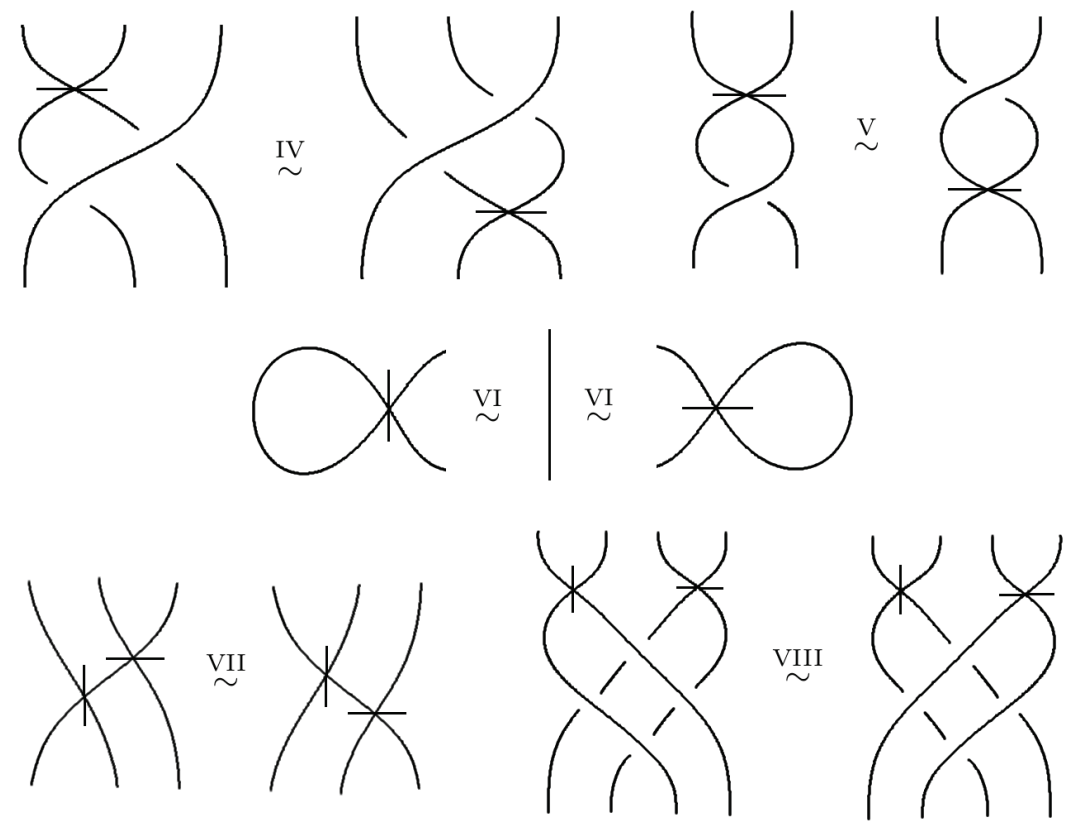

See, for instance, $[\mathbf{5}, \mathbf{1 4}, \mathbf{1 5}, \mathbf{1 6}, \mathbf{1 7}]$ for more.

In [20], bikei colorings and counting invariants of marked graph diagrams were considered. Specifically, all of the semiarcs meeting at a saddle crossing determine the same generator of $\mathcal{B K}(\Sigma)$ and must have the same color. We now observe that we can enhance the bikei counting invariant with bikei 2-cocycles in the same way as we did for knots and links in $\mathbb{R}^{3}$. Specifically, we have

Definition 5.1. Let $X$ be a finite bikei and $\phi \in H_{B K}^{2}(X)$. Then for any unoriented knotted surface $\Sigma$ represented by a marked vertex diagram $D$, the 2-cocycle enhanced 
bikei counting invariant of $\Sigma$ is the multiset

$$
\Phi_{X}^{\phi, M}(D)=\left\{B W\left(D_{f}\right) \mid f \in \operatorname{Hom}(\mathcal{B K}(\Sigma), X)\right\},
$$

or its generating function

$$
\Phi_{X}^{\phi}(D)=\sum_{f \in \operatorname{Hom}(\mathcal{B K}(\Sigma), X)} u^{B W\left(D_{f}\right)} .
$$

We then have

Theorem 5.2. If $X$ is a finite bikei, $\phi \in H_{B K}^{2}(X)$ and $D$ and $D^{\prime}$ are unoriented marked vertex diagrams related by Yoshikawa moves, then

$$
\Phi_{X}^{\phi, M}(D)=\Phi_{X}^{\phi, M}(D) \quad \text { and } \quad \Phi_{X}^{\phi}(D)=\Phi_{X}^{\phi}(D) .
$$

Proof. This is a matter of verifying that the Yoshikawa moves do not change the Boltzmann weight of a bikei colored marked vertex diagram. Moves VI and VII do not involve non-saddle crossings, so these cannot change the Boltzmann weight, and in move $\mathrm{V}$ all semiarc colors are the same, so both sides of the move contribute $\phi(x, x)=0$. For moves IV and VIII, both sides of the move contribute degenerate chains: $\phi(x, y)+\phi(x, y \bar{*} x)$ on both sides of move IV, and $2 \phi(y, x)+2 \phi(y, x \bar{*} y)$ on the left and $2 \phi(x, y)+2 \phi(x, y \bar{*} x)$ on the right of move VIII.
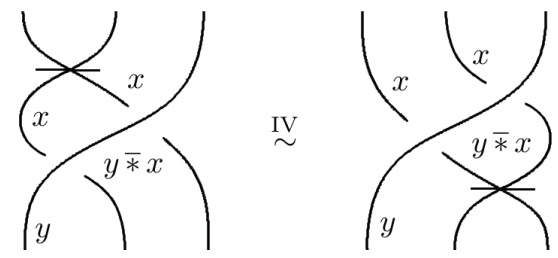
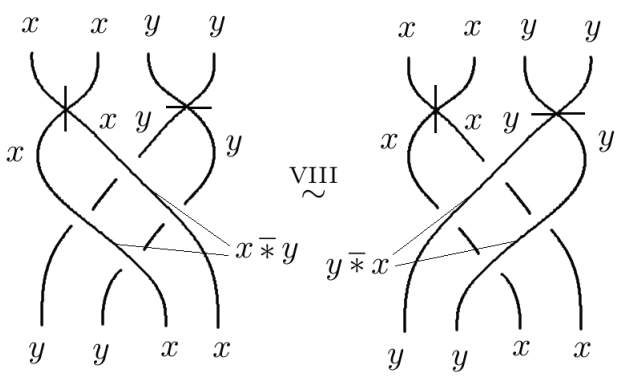

Analogously to the case of knotted and linked curves, including virtual crossings in marked vertex diagrams yields virtual knotted surface diagrams, with the rule that two such diagrams are equivalent if related by Reidemeister moves, Yoshikawa moves and the detour move, i.e., redrawing an arc with only virtual crossings as another arc with only virtual crossings and the same endpoints. See [13] for more.

Example 5.3. Let $X$ again be the Alexander bikei from Example 3.8 and consider the virtual marked vertex diagram $D$ below, representing a virtual linked surface with one sphere component and one projective plane component.

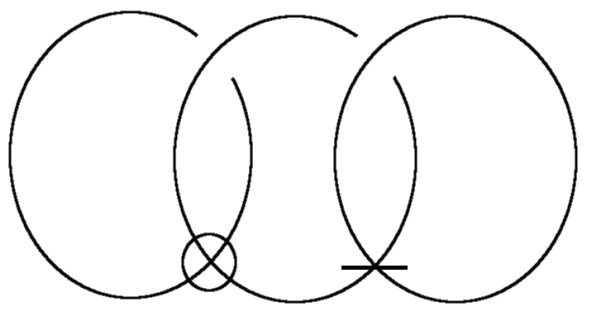


$X$-labelings of $D$ are given by pairs $(x, y) \in\left(\mathbb{Z}_{8}\right)^{2}$ satisfying $3 y=y$, i.e., $2 y=0$, with $x=x+2 y$ imposing no further conditions on $x$ :

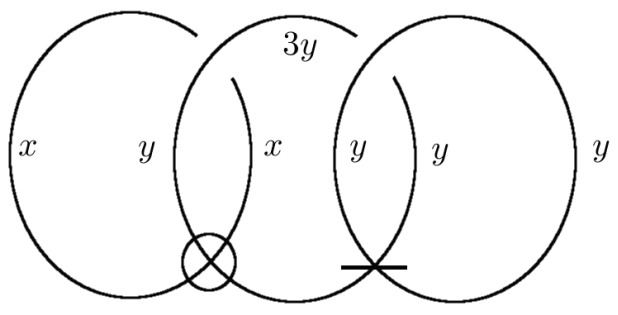

Hence we have $16 X$-colorings as in Example 4.3; each coloring has Boltzmann weight $4(x-y)+4(y-y)$, so colorings with odd $x$ contribute $u^{4}$ while colorings with even $x$ contribute 1 to the invariant, and we again have $\Phi_{X}^{\phi}(K)=8+8 u^{4}$.

\section{Questions}

We conclude with a few questions for future research.

How does bikei homology generalize to other cases like involutory biracks, virtual bikei and parity bikei? What about shadow colorings, either in the marked vertex style or broken surface diagram style? It seems that additional degeneracies might be required for shadow colorings, depending on the shadow coloring operation; what should these be?

We propose the following

Conjecture 6.1. The free part of $H_{B K}^{2}(X ; \mathbb{Z})$ is $\{0\}$ for all finite bikei $X$.

Is this conjecture true? If not, what is the smallest counterexample?

\section{References}

[1] S. Aksoy and S. Nelson. Bikei, involutory biracks and unoriented link invariants. J. Knot Theory Ramifications, 21(6):1250045, 13, 2012.

[2] J.S. Carter, M. Elhamdadi, and M. Saito. Homology theory for the set-theoretic Yang-Baxter equation and knot invariants from generalizations of quandles. Fund. Math., 184:31-54, 2004.

[3] J.S. Carter, D. Jelsovsky, S. Kamada, L. Langford, and M. Saito. Quandle cohomology and state-sum invariants of knotted curves and surfaces. Trans. Amer. Math. Soc., 355(10):3947-3989, 2003.

[4] J.S. Carter, S. Kamada, and M. Saito. Stable equivalence of knots on surfaces and virtual knot cobordisms. J. Knot Theory Ramifications, 11(3):311-322, 2002. Knots 2000 Korea, Vol. 1 (Yongpyong).

[5] S. Carter, S. Kamada, and M. Saito. Surfaces in 4-Space, volume 142 of Encyclopaedia Math. Sci. Springer-Verlag, Berlin, 2004. Low-Dimensional Topology, III.

[6] J. Ceniceros, M. Elhamdadi, M. Green, and S. Nelson. Augmented biracks and their homology. Internat. J. Math., 25(9):1450087, 19, 2014. 
[7] M. Elhamdadi and S. Nelson. $N$-degeneracy in rack homology and link invariants. Hiroshima Math. J., 42(1):127-142, 2012.

[8] M. Elhamdadi and S. Nelson. Quandles-An Introduction to the Algebra of Knots, volume 74 of Stud. Math. Libr. American Mathematical Society, Providence, RI, 2015.

[9] R. Fenn and C. Rourke. Racks and links in codimension two. J. Knot Theory Ramifications, 1(4):343-406, 1992.

[10] R. Fenn, C. Rourke, and B. Sanderson. Trunks and classifying spaces. Appl. Categ. Structures, 3(4):321-356, 1995.

[11] D. Joyce. A classifying invariant of knots, the knot quandle. J. Pure Appl. Algebra, 23(1):37-65, 1982.

[12] L.H. Kauffman. Virtual knot theory. European J. Combin., 20(7):663-690, 1999.

[13] L.H. Kauffman. Virtual knot cobordism. In New Ideas in Low Dimensional Topology, volume 56 of Ser. Knots Everything, pages 335-377. World Sci. Publ., Hackensack, NJ, 2015.

[14] J. Kim, Y. Joung, and S.Y. Lee. On the Alexander biquandles of oriented surface-links via marked graph diagrams. J. Knot Theory Ramifications, 23(7):1460007, 26, 2014.

[15] J. Kim, Y. Joung, and S.Y. Lee. On generating sets of Yoshikawa moves for marked graph diagrams of surface-links. J. Knot Theory Ramifications, 24(4):1550018, 21, 2015.

[16] S.Y. Lee. Towards invariants of surfaces in 4-space via classical link invariants. Trans. Amer. Math. Soc., 361(1):237-265, 2009.

[17] S.J. Lomonaco, Jr. The homotopy groups of knots. I. How to compute the algebraic 2-type. Pacific J. Math., 95(2):349-390, 1981.

[18] S.V. Matveev. Distributive groupoids in knot theory. Mat. Sb. (N. S.), 119(161)(1):78-88, 160, 1982.

[19] T. Mochizuki. Some calculations of cohomology groups of finite Alexander quandles. J. Pure Appl. Algebra, 179(3):287-330, 2003.

[20] S. Nelson and P. Rivera. Bikei invariants and gauss diagrams for virtual knotted surfaces. J. Knot Theory Ramifications, 25(3):1640008, 14, 2016.

[21] S. Nelson and J. Vo. Matrices and finite biquandles. Homology Homotopy Appl., 8(2):51-73, 2006.

[22] M. Takasaki. Abstraction of symmetric transformations. Tohoku Math. J., 49:145-207, 1943.

Sam Nelson Sam.Nelson@cmc.edu

Jake Rosenfield jlrosenfield@gmail.com

Department of Mathematical Sciences, Claremont McKenna College, 850 Columbia Ave., Claremont, CA 91711, USA 